\title{
A Novel Approach for Generating and Optimizing Electricity by using Runway Exhaust
}

\author{
Sumit Das \\ Department of Information \\ Technology \\ JIS College of Engineering, \\ Kalyani, India
}

\author{
Manas Kumar Sanyal, PhD \\ Department of Business \\ Administration \\ University of Kalyani \\ Kalyani, India
}

\author{
Debamoy Datta \\ Department of Electrical \\ Engineering \\ JIS College of Engineering, Un \\ Kalyani, India
}

\begin{abstract}
Presently the world is running through an energy crisis and the utilization of the renewable resources is still under development. The present challenge in this era is to use the waste energy that can generate ample amount of electricity, like in industries for maintaining draught of the flue gases, certain amount of energy is just thrown out into the atmosphere by the chimneys to keep the system running. Similarly, the hot gases thrown away by the aircraft also goes waste, so in this way there are lot of sources of waste energy, that if utilized properly would eliminate the need of fossil fuels like coal. In addition, if devices utilizing the energy of these systems were integrated in the micro-grid architecture, the efficiency as well as reliability of the power system would increase. This paper develops a method to utilize the waste energy from aircrafts in the runway to generate electric energy. The work also estimates the optimum number of units, power that is to be used to achieve maximum profit, which is analyzed using Genetic Algorithm, an optimum method of placing the units is developed. A control system representation of the entire system is developed, and they are simulated against various types of expected input response. Apart from the control system block diagram mathematical model of jet blast velocity is developed. The formula for efficiency of the bladeless wind turbine is also developed.
\end{abstract}

\section{General Terms}

Renewable Energy: Wind Energy to electrical energy.

\section{Keywords}

Jet blast, Bladeless wind energy generator, piezoelectric material, Genetic Algorithm

\section{INTRODUCTION}

The demand for energy from renewable resources are growing day by day, The main focus for few decades in this area had been in the area of solar panel, bladeless wind turbine, development of microgrid structure but none of the papers that are available today focus on the waste energy i.e. the energy that it cannot harness ordinarily, for example the waste gases that gets escaped from chimney, thermodynamics does not allow us to do that but apart from the above mentioned scenario there are few cases where we can utilize this energy, in this paper authors propose and analyze such a system that is able to harness waste energy efficiently. Whenever there is any aircraft takeoff or landing near a runway a huge amount of hot gases escape through the jet engines and passes through the runway carrying a huge amount of energy, this energy is essentially wasted so in this paper we propose a methodology to harness this energy, which can save significant amount of money. A runway consists of many different types of lighting system focus is on the edge lights, which are placed 60 meters apart; we utilize this space efficiently to place the wind generators. Firstly, in background study we survey some literature regarding the problems that presently power system faces. Then the literature survey is also done on the present problems that the utilization of renewable resources has, following which we describe the method of working in the methodology section, following which a description of control system block diagram of the overall architecture is made and simulations done in MATLAB. Following which we derive the efficiency of the system and then provide the economic savings that the system can provide by using the Genetic algorithm.

\section{LITRERATURE REVIEW}

Various works are done on the field of harnessing renewable energy $[8,9]$, the main advantage of this renewable energy system is that with the help of micro grid system we can store the energy and in case of power outages use them for fulfilling the needs. In a paper by Md. MoyeedAbrar [1] which was published in 2016 stated few challenges that India's power sector is currently facing these are:

a) Increasing growth in demand for power and the nation's fast economic growth and rising living standards.

b) Lack of uninterrupted and continuous power supply in rural and backward regions. The electricity from the national grids reaches over $93 \%$ of urban areas but more than half of rural population has no access to electricity

c) Malfunctioning of the electricity grids. Distribution line losses in India are rated to be among highest in the World, averaging over $30 \%$. The northwestern regions of the country and rural areas are dented quite badly.

d) Theft of electricity: Electricity theft is a major issue in all parts of the country.

We list here only four as the main concern is point $1,2 \& 3$ as with the advent of digital meters theft has reduced considerably. A simple solution to the above problem may seem solar PV cells storing energy in inverters but there are disadvantages for them also, in a paper by Suparva Chakraborty, Rajesh Kumar and others[2] a mathematical model for finding the best suited PV technology for different climatic zones of India is proposed wherein. Global horizontal insolation $(\mathrm{GHI})$ and daytime temperature are the two major parameters affecting the output of photovoltaic (PV) plant and thus it cannot say that they will always produce the desired amount of energy every time. Therefore, what comes to the mind next is wind energy that is like solar energy freely available. Still wind power is dependent upon the weather conditions. In a paper by Chen, Wang [3] this paper proposed a new dispatch method for wind farm (WF) 
cluster by considering wind power forecasting errors. So a major problem is to remove the dependency of climate. In this paper, authors propose a Wind Power system that is free from variation of climate. After the review of literature it can be concluded that at present the use of renewable resource is both time consuming as well as unpredictable as predefined calculation are to be made for their placement. Climatic factors are to be considered, still the amount of power that one can receive is probabilistic and to address these problems, and propose a bladeless wind power generation technique that utilizes the waste energy in the form of jet blast and hypothesize that the system proposed is stable as well as economic in nature.

\section{METHODOLOGIES}

As is clear from the figure 1 that whenever there is any aircraft that lands or takes off from runway, a strong flow of hot gases that comes from the jet engine of the plane, as a result near the edge of the runway there is always flow of air because aircrafts taking off the runway and landing.

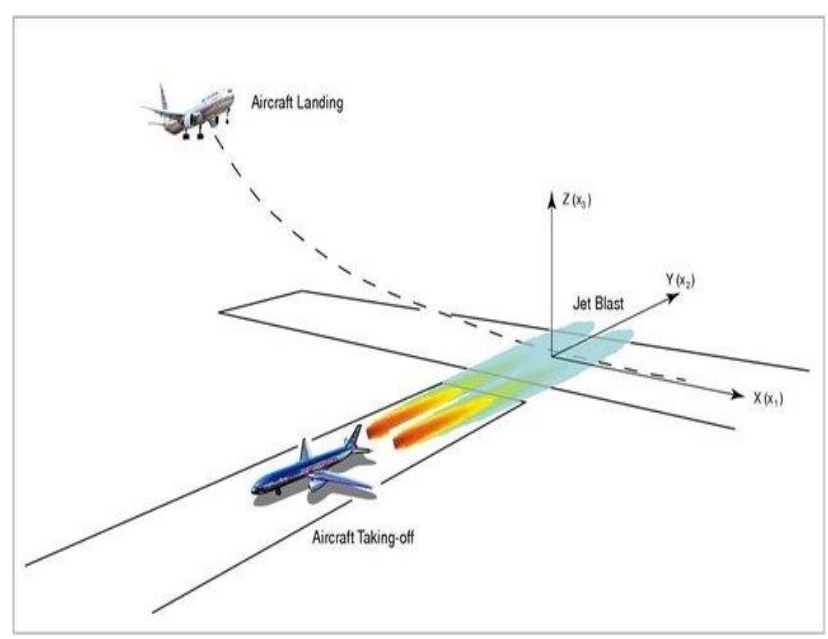

Figure 1: showing the jet blast from the runway [5]

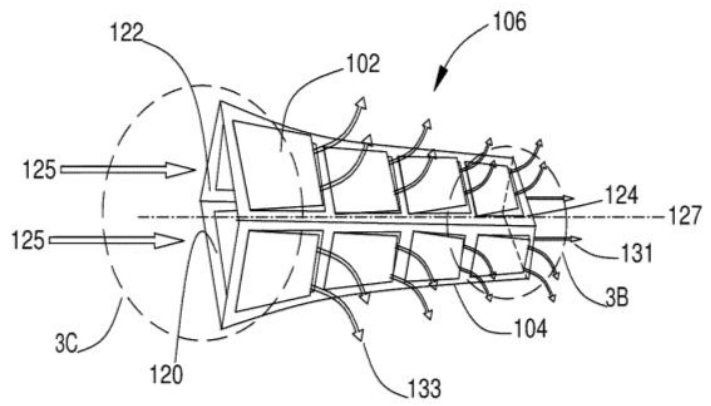

Figure 2: Diagram of bladeless wind power turbine [4].

The above figure 2 shows the schematic diagram of bladeless wind power generator which was patented in 2010 [4]. The following are the features of this design:

a) Application of small air jet tunnels with side cantilever arrays;

b) Incorporation of a PVDF-brass-PVDF sandwich piezoelectric cantilevers as kinetic-electric conversion devices;

c) Integration of a cascading phononic structure specifically designed and constructed for an air jet tunnel and sandwich cantilever array.
In simplest description, when air passes through the air jet tunnel, it makes the cantilever vibrate as this is connected to a piezoelectric material, these vibrations produce electrical energy. The main advantage of the proposed methodology is that even if this technology is used in verycold climates, the hot air from the jet engines will prevent ice from depositing in those jet tunnels. The generated power as given by them [4] are:

Wind panel Power:

$\mathrm{P}=0.5 \times \mathrm{D}_{\mathrm{A}} \times \mathrm{A} \times \mathrm{Cp} \times \mathrm{V}^{3} \times \mathrm{C}_{\mathrm{E}}$

where: $\mathrm{P}=$ power in watts; $\mathrm{D}_{\mathrm{A}}=$ air density (about 1.225 $\mathrm{kg} / \mathrm{m}^{3}$ at sea level, less higher up); $\mathrm{A}=$ panel area, exposed to the wind $\left(\mathrm{m}^{2}\right) ; \mathrm{Cp}=$ Coefficient of performance $(0.59$ \{ Betz limit $\}$ is the maximum theoretically possible, 0.5 for a good design of air jet tunnel); V=wind speed in meters/sec; $\mathrm{C}_{\mathrm{E}}=$ Conversion efficiency of the cantilever array panel (the estimated value of WEP is 50\%). With a good design, this equation can be expressed as:

$P / A=0.5 \times 1.225 \times 0.5 \times V^{3} \times C_{\mathrm{E}}$

If it wants a $3 \mathrm{~kW}$ generator with a wind resource of 10 meters per second (32.81 feet/second) and 50\% efficiency $\left(C_{E}\right)$, the panel area "A" should be $3000 / 153=19.6 \mathrm{~m}^{2}$ that will require a volume of 2 cubic meters (2.616 cubic yards) because the thickness of the WEP panel is only $10 \mathrm{~cm}(3.84$ inches).
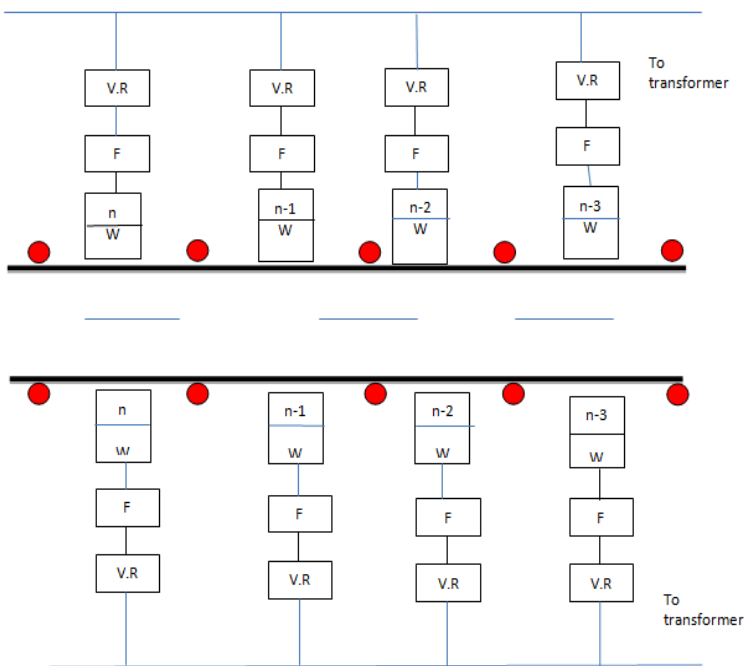

Figure 3a: Showing the single line diagram representation of the system proposed

Table 1: The table of symbols for the single line diagram

\begin{tabular}{|c|c}
\hline \multicolumn{1}{|c|}{ Symbols } & \multicolumn{1}{c}{ Description } \\
\hline Th & $\begin{array}{l}\text { This represents n number of wind power generators connected } \\
\text { in series and separated by isolating transformers. The upper } \\
\text { number represents the number of units. } \\
\text { This represents the frequency changing circuit panel }\end{array}$ \\
\hline V.R & $\begin{array}{l}\text { This represents the voltage regulating circuit panel } \\
\text { This represents the centerline lights } \\
\text { This represents the edge lights }\end{array}$ \\
\hline
\end{tabular}


After the power is generated in the wind power generators the supply is fed to frequency changers[6] that changes the frequency of the generated voltage to $50 \mathrm{~Hz}$ following which they are fed to the voltage regulator circuits after that they are sent to the isolating transformers then to the main transformer that are connected to the bus bars.

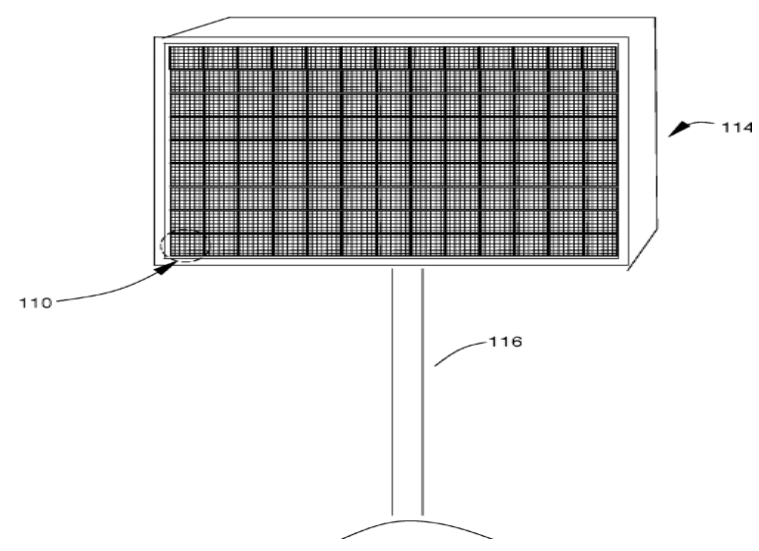

Figure 3b: This is the single generating unit as seen from side view

This is the diagram of wind power generating unit, additional supports may be provided to the mechanical structure. Many such modules are used in the system to generate the power, As is clear from the figure 8 that no moving parts are involved hence these structure can tolerate the high velocity winds from the jet blast.

The following subsection represents the whole control system and its simulation.

\subsection{Wind Velocity to mechanical displacement conversion}

Now as the jet blast passes through the device, the wind velocity is converted into simple harmonic displacements of the piezoelectric crystal. Let us call this process as mechanical converter. The input in this case is wind velocity and the output in this case is displacement that get across the piezoelectric crystal.

Let $V_{j e t}$ be the velocity of air in jet blast, the time for which this velocity remains is from "a" seconds to "b" seconds. So the input function can be modeled as:

$V(t)=V_{\text {jet }}(u(t-a)-u(t-b))$

The corresponding output that is the displacement $x(t)$ that varies sinusoidal in time. Therefore, the corresponding output function is:

$x(t)=x_{A} \sin (\omega t)$

The Laplace transform of the equation (2) becomes,

$V(s)=V_{j e t}\left(\frac{e^{-a s}}{s}-\frac{e^{-b s}}{s}\right)$

Similarly the equation 3 becomes:

$X(s)=\frac{x_{A} \omega}{s^{2}+\omega^{2}}$

So dividing equation 5 by 4 and simplifying, the transfer function becomes:

$$
G_{1}(s)=\frac{x_{A}}{V_{j e t}}\left(\frac{\omega s}{\left(s^{2}+\omega^{2}\right)\left(e^{-a s}-e^{-b s}\right)}\right)
$$

Now, for small values of $s$, the exponential terms can be approximated as:

$$
e^{-a s} \approx\left(1-a s+\frac{a^{2} s^{2}}{2}\right)=\left(1-a s+0.5 a^{2} s^{2}\right)
$$

$\left.0.5 b^{2} s^{2}\right)$

$$
e^{-b s} \approx(1-b s+
$$

Putting the above two relations and simplifying the transfer function:

$G_{1}(s)$

$\approx \frac{x_{A}}{V_{\text {jet }}}\left(\frac{\omega s}{\left(s^{2}+\omega^{2}\right)\left(s(b-a)+0.5 s^{2}\left(b^{2}-a^{2}\right)\right)}\right)$

\subsection{Simplified piezoelectric Generator model for the bladeless wind power generator}

In a piezoelectric crystal the output voltage is given by:

$$
V_{\text {piezo }}=h_{11} \Delta x
$$

Or, equivalently speaking:

$$
V_{\text {piezo }}=h_{11} d x
$$

But in this case the displacement is sinusoidal varying so, taking differential of equation (3)

$$
V_{\text {piezo }}=h_{11} x_{A} \cos (\omega t) d t
$$

Assuming dt to be 1 ,

$V_{\text {piezo }}(t)=h_{11} x_{A} \cos (\omega t)$

Taking the Laplace transform of (7)

$V_{\text {piezo }}(s)=\frac{h_{11} x_{A} \mathrm{~s}}{\mathrm{~s}^{2}+\omega^{2}}$

Dividing equation (8) by the equation (5), as displacement is the input, we get after simplification:

$G_{2}(s)=\frac{h_{11} s}{\omega}$

\subsection{Simplified Transfer function of} Frequency changer present in the generator Now, the working of frequency changer can be shown as:

$$
\begin{aligned}
& v(t) \\
& \rightarrow v\left(\frac{1}{a} t\right)
\end{aligned}
$$

Now, both input as well as output is sinusoidal in nature with just a change of scale, So after putting $v(t)=V_{A} \sin (\omega t)$ and using equation (10) the transfer function becomes:

$$
\begin{aligned}
& G_{3}(s) \\
& =\frac{\left(s^{2}+\omega^{2}\right)}{a s^{2}+\omega^{2}}
\end{aligned}
$$




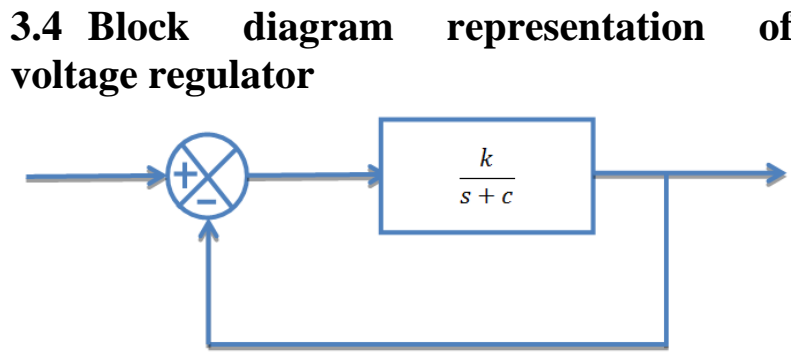

Figure 4: Block diagram representation of the voltage regulator

This is the simplified block diagram representation of the voltage regulator, here unity feedback is used, as the error voltage passes through the block, it is reduced exponentially :

$$
\varepsilon(t)=e^{-c t} \varepsilon
$$

Where, $\varepsilon$ is the steady state error that get after unity feedback. The transfer function represented in the block can be implemented as a RC circuit.

\subsection{Overall block diagram of the entire system}

In the first step the strong jet blast strikes the panels of the wind power generator that converts the velocity of wind to sinusoidal mechanical vibrations which is represented in the transfer function block diagram of figure 5 by $G_{1}(s)$

Then theses vibrations are applied to the piezoelectric material which converts the sinusoidal displacements to sinusoidal voltage represented in the block diagram of the figure 5 by the transfer function $G_{2}(s)$. Then the frequency needs to be changed to $50 \mathrm{~Hz}$ so that it can be fed to the bus bars, this is done by frequency changer and is represented in the block diagram of figure 5 by the transfer function $G_{3}(s)$. Then this voltage is fed to the voltage regulator circuit that prevents sudden voltage surges and is represented by the last block diagram with the unity feedback.

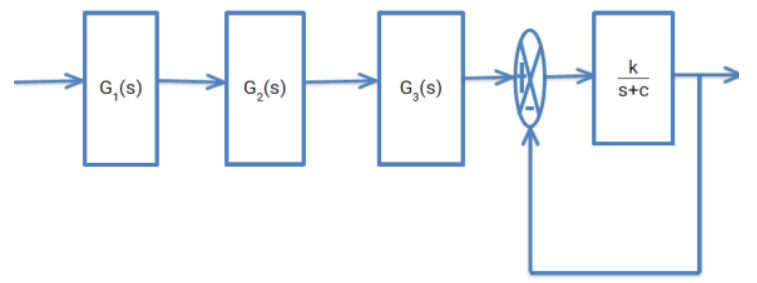

Figure 5: The overall block diagram representations of the system.

Table 2. Table of description for block diagram

\begin{tabular}{cl}
\hline $\begin{array}{l}\text { Names of transfer function } \\
\text { in Block diagram }\end{array}$ & Description \\
\hline$G_{1}(s)$ & $\begin{array}{l}\text { Wind velocity to mechanical } \\
\text { displacement converter }\end{array}$ \\
$G_{2}(s)$ & Piezoelectric converter \\
$G_{3}(s)$ & Frequency changer \\
$G_{4}(s)$ & $\begin{array}{l}\text { Voltage regulator having open } \\
\text { loop transfer function } \\
\end{array}$ \\
& incorporated by unity feedback \\
\hline
\end{tabular}

\subsection{Calculation of efficiency of the system}

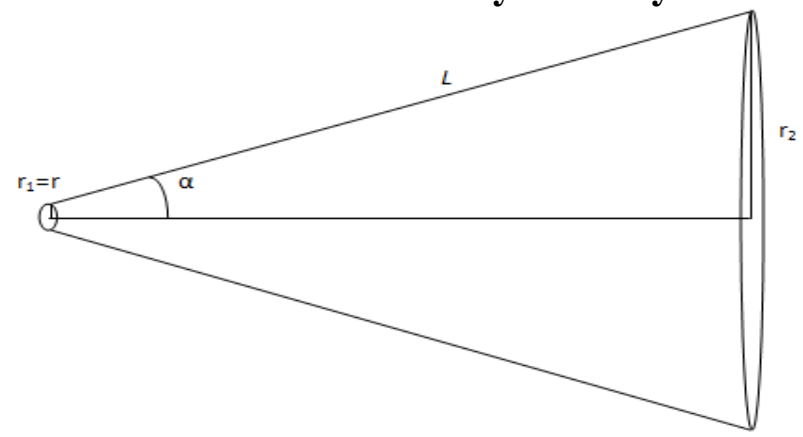

Figure 11: Simplified model of Jet engine

The above figure 11 shows the control volume surrounding the jet engine. Consider a control volume in the shape of the jet engine as shown in the figure 11 , in the analysis, and assume that the hot air from the jet engines mainly contribute to the jet blast, with this simplifying assumption, we apply the mass balance equation to the control volume.

$$
\dot{m}_{1}+\dot{m}_{1}^{\prime}=\dot{m}_{2}
$$

Where the $\dot{m}_{1}$ is the inlet mass flow rate, $\dot{m}_{1}^{\prime}$ is the mass that is injected, assume that the injected mass is negligible as compared to the inlet mass flow and $\dot{m}_{2}$ is exit mass flow rate.

So, the above equations become:

$$
\rho_{0} A_{i} V_{i}=\rho_{f} A_{f} V_{j e t}
$$

Now, from the ideal gas equation:

$$
P=\rho_{f} R T_{\text {hotgases }}
$$

Putting the value of $\rho_{f}$ in equation (12) and by geometry of the figure (11) with $r_{2}=l \sin \alpha$, simplify the above equations and get :

$$
V_{j e t}=\frac{\rho_{0} R T_{\text {hotgases }} r^{2} V_{i}}{p l^{2} \sin ^{2} \alpha}
$$

Now, substituting the value of equation (14) in equation (1):

$$
P=0.5 \times \mathrm{D} \mathrm{A} \times \mathrm{A} \times \mathrm{Cp} \times \mathrm{k}^{3} \mathrm{~T}_{\text {hot gases }}^{3} \times \mathrm{CE}
$$

Where, $\mathrm{K}=\frac{\rho_{0} R r^{2} V_{i}}{p l^{2} \sin ^{2} \alpha}$

Or, $T_{\text {hotgases }}=(k 2) P^{\frac{1}{3}}$

i.e. Power generated is proportional to the cube of the temperature of the hot gases. The temperature that the system gets is lowered due to surroundings. Therefore, the thermal efficiency of the system can be taken as actually the measure of its efficiency, so

$\eta=\frac{T_{\text {hotgases }}-T_{\text {surronding }}}{T_{\text {hotgases }}}$

\subsection{Evaluation of Economic cost saving by this generator using Genetic Algorithm}

Maximum numbers of units are placed in the position where there is maximum jet blast and the number of units are decreased by 1 for every combination of units that are placed 
certain distance apart covering the runway because jet blast is strongest at starting and its strength decreases by small amounts along the runway. i.e. for example in the region where there is maximum amount of jet blast, $n$ units of generators are placed then after a certain distance (n-1) unit of generators are placed and so on. Commercially, it costs Rs. 2 per unit or per kilowatt-hour then by unitary method.

$$
P .(1 \text { hour }) \rightarrow R s \cdot \frac{2 P}{1000}
$$

As $\mathrm{P}$ is in watts, so divide it by 1000, So Rs. $2 P / 1000$ can be saved for every unit of electricity that is generated. We describe the cost function as:

$$
Z(n)=\sum_{i=1}^{n} \frac{2 P i}{1000}
$$

Since each unit saves this amount of cost, so the costs by the individual units add up, further the individual power generated by each unit is constant. Simplifying the above equation, we get:

$$
Z(n)=\frac{2 P}{1000}\left(\frac{n(n+1)}{2}\right)
$$

Or, $Z(n)=\frac{P}{1000}(n(n+1))$

Subjected to the constraints,

$$
P n \leq \alpha
$$

Where $\alpha$ is the maximum load demand. Now, as the power and load demand fluctuates, $\mathrm{n}$ must not increase suddenly but at a slow rate and steadily and then increase linearly for stable operation and to ensure that voltage surges are decreased. So:

$$
\frac{\Delta n}{\Delta p}=n\left(1-e^{-a n}\right)
$$

But this should also be within some limits i.e. at most this change can be $\beta$. So the above equation can be written as:

$$
n\left(1-e^{-a n}\right) \leq \beta
$$

So the problem is to optimize the function described by the equation (17) subjected to constraints (18) and (19). We have to use Genetic algorithm to optimize the problem as it involves, nonlinear constraints. $\mathrm{R}$ language was used for this [7].The formal definition of the problem is:

Problem: maximize $Z(n)=\frac{P}{1000}(n(n+1))$

Subjected to constraints: $P n \leq \alpha$ and $n\left(1-e^{-a n}\right) \leq \beta$ where $\alpha$ and $\beta$ are taken as 1000 and 200 respectively plot of cost saved-vs- power and number of units

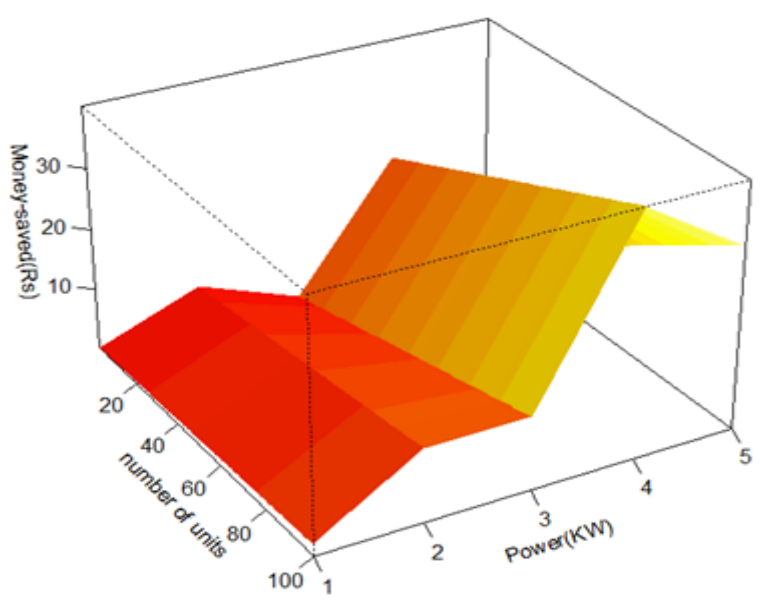

Figure 12: Visualization of the objective function in 3 dimensions

The figure 12 is the plot of the objective function which is the money saved against the independent variables the number of units and power generated by a particular unit, the plot shows that the function has steep slopes at certain points,

so finding an optimal solution is difficult, genetic algorithm can alone give the best optimal solution. The fitness function is defined as:

$$
\begin{aligned}
& \text { Fitness = objectivefunc } \left.-\left(\max \left(\left(P_{n}-a\right)\right), 0\right) * \text { large_number }\right)-\left(\max \left(\left(n\left(1-e^{-n}\right)-\beta\right), 0\right) *\right. \\
& \text { large_number })
\end{aligned}
$$

Whereas is clear from the equations (17), (18) and (19) that this is a multivariable optimization.The objective function in this case is basically equation (17).In order to incorporate for the constraints in the equations (18) and (19), the two terms apart from objective function are incorporated in the fitness function.consider the first term if $P n \leq \alpha$ then $P n-\alpha \leq 0$. So the maximum of 0 and $P n-\alpha$ is 0 , so the term $(\max ((P n-\alpha)), 0) *$ large_number $)$ becomes zero and there is no effect on the fitness value, whereas if the contrained is not satisfied $(\max ((P n-\alpha)), 0) *$ large_number)term becomes very large and fitness for the population decreases very much, similar phenomena occurs with the other term in the fitness function. Now if the value of the objective function increases to maximum value then the fitness of the population also increases, thus the genetic algorithm optimizes the problem. The results are described in the following sections. 


\section{RESULT AND ANALYSIS}

\subsection{Results of the control system simulation}

The MATLAB has been used for simulation of this control system and the results are as follows.

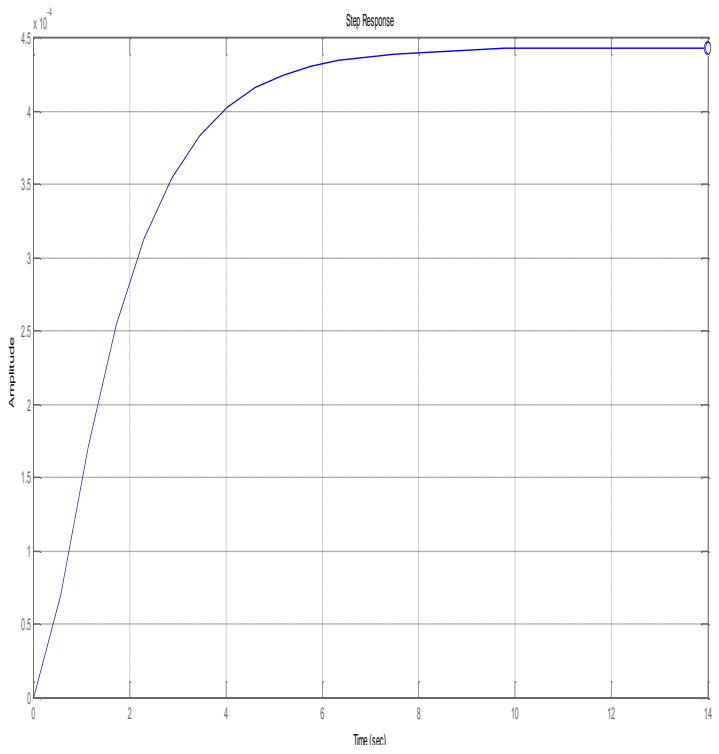

Figure 6: step response of the overall transfer function, $\omega=52 \mathrm{~Hz}$ a and $b$ for transfer function are 1 and 2 respectively

After making the entire block diagram in MATLAB, we gave the step input, now while making the simulation, the parameters needed to be given values. The physical significance of these parameters for this transfer function which is G1(s) are, a is the start time and b is the stop time of the jet blast which should produce the desired sinusoidal output as derived in previous sections, actually these parameters are design parameters. Now in the figure 6 , we actually have a very short period of jet blast for which sinusoidal output is expected, but from the results, observe that output is constant stable dc but directly want Alternating current for which these parameters of $a$ and $b$ should not be used and the results confirm that.

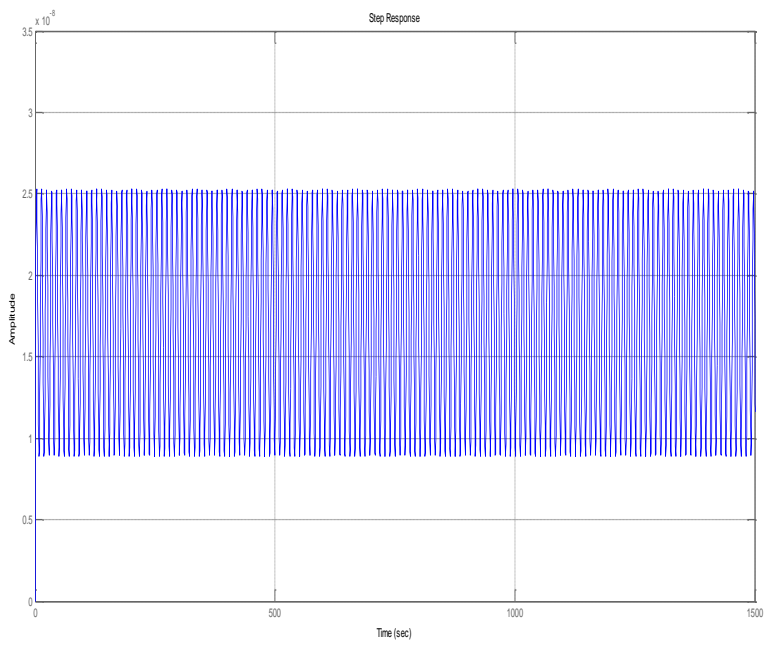

Figure 7: step response of the overall system when a is 0 and $b$ is 100 in G1(s).
On the other hand consider the figure 7; here the wind velocity to mechanical displacement converter is designed such that for jet blast lasting for 100 seconds sinusoidal output is produced. So, for designing the system, during its manufacture the parameters $a$ and $b$ needs to be correctly set as these depends only on the mechanical design.

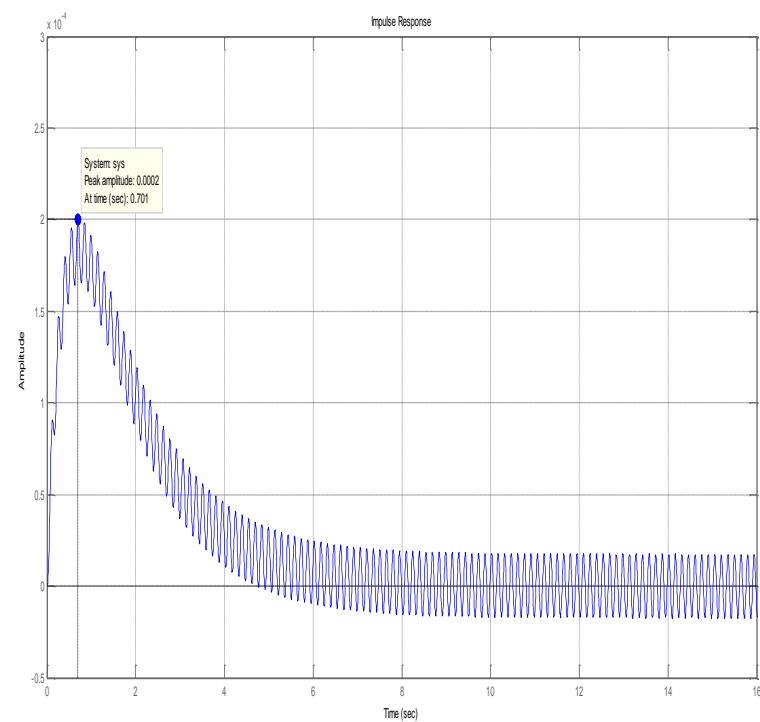

Figure 8: impulse response when $\mathrm{a}$ in the transfer function G3 is $\mathbf{1 . 5}$

Now, the distinction that make here is that parameters in transfer function are all design parameters. So, with the proper values of design parameters for wind velocity to mechanical displacement converter which, get from figure 7 we apply the input but for the figure 8 and 9 the parameters for figure 6 are used, upon giving impulse input or step input we should get stable oscillations and that is what we get, noting that for step input this was not possible. The figure 8 confirms the result that impulse input produces the stable oscillations. Now the point of interest here is the design parameter $\mathrm{a}$ in the frequency changer. Here in figure 8 the parameter is kept as 1.5 .

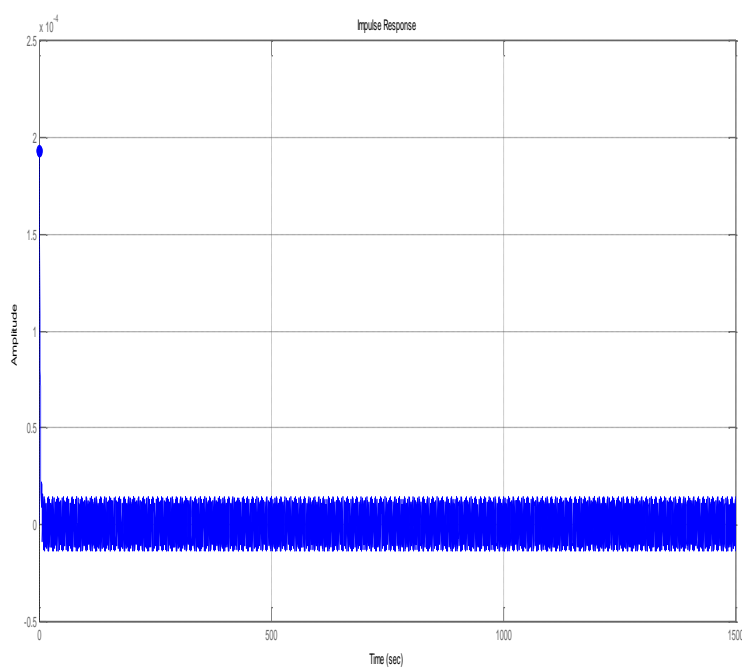

Figure 9: impulse response when $a$ in the transfer function, G3(s) is 1

In the same system all the parameters are kept constant and only the design parameter a is decreased by 0.5 , the result we see the frequency has increased considerably but the 
overshoot is same but the period for which it stays there is reduced.

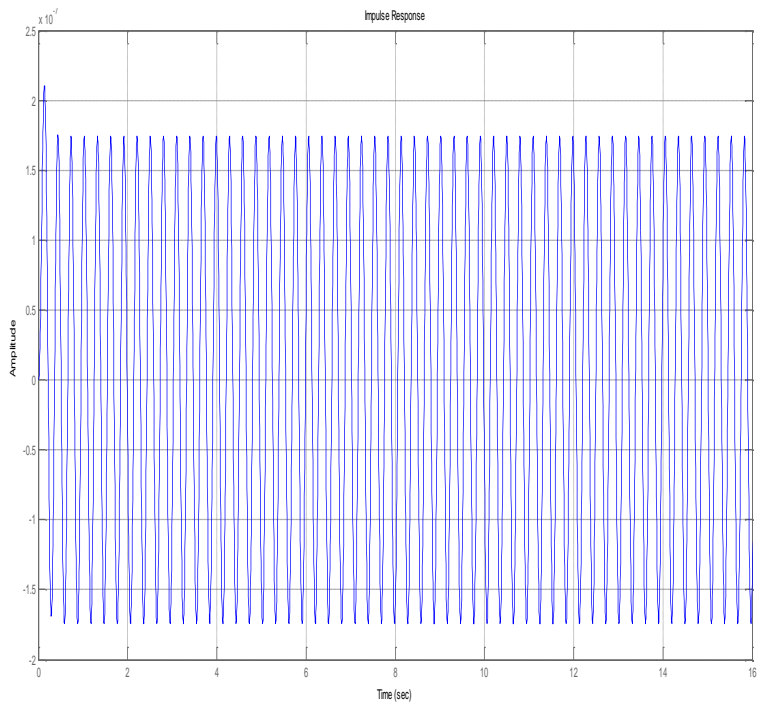

Figure 10: impulse response of the system when $b$ is 100 and $a$ is 0 for the transfer function G1(s)

Same thing is shown in figure 10, just proper design parameters of figure 7 and 8 are used to run the system with impulse input, the result is an ideal sinusoidal wave. The fact here is that normally jet blast is for very short period and we can assume that input will be sinusoidal. Higher order harmonics are only produced with step input which assumes that the jet blast is there forever which is hypothetical but overall such harmonics can be reduced by adjusting the design parameter a for the system.

\subsection{Results of genetic Algorithm for economic cost saving}

On running the algorithm in $\mathrm{R}$, we get a plot of the fitness value against the number of generations as shown in the figure 13, next to know all the parameters as well as the final result we call the summary function in $\mathrm{R}$ as is depicted in figure 14. The best value of the fitness function should be 0 which is shown by a green line, however due to mutations, this value is never achieved, Median is the fitness value that is reached maximum number of times ,it is shown by light green area, the mean value of fitness for that generation is shown by blue line.

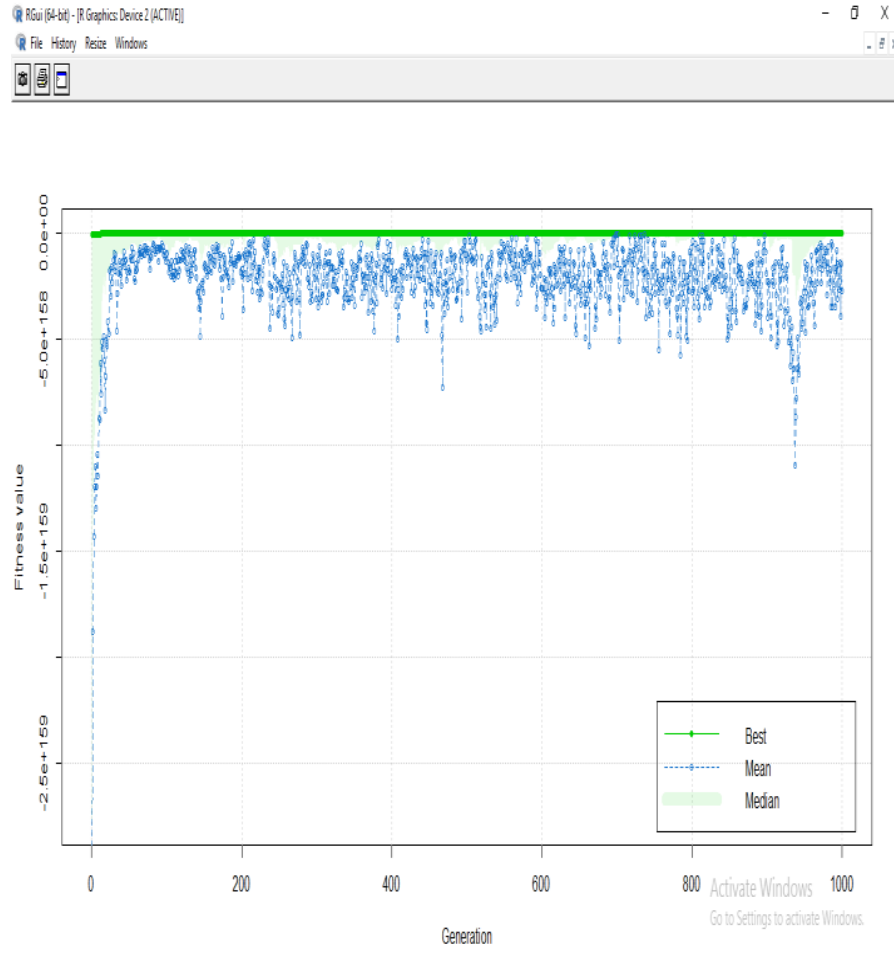

Figure 13: Fitness value plot against the number of generation of population

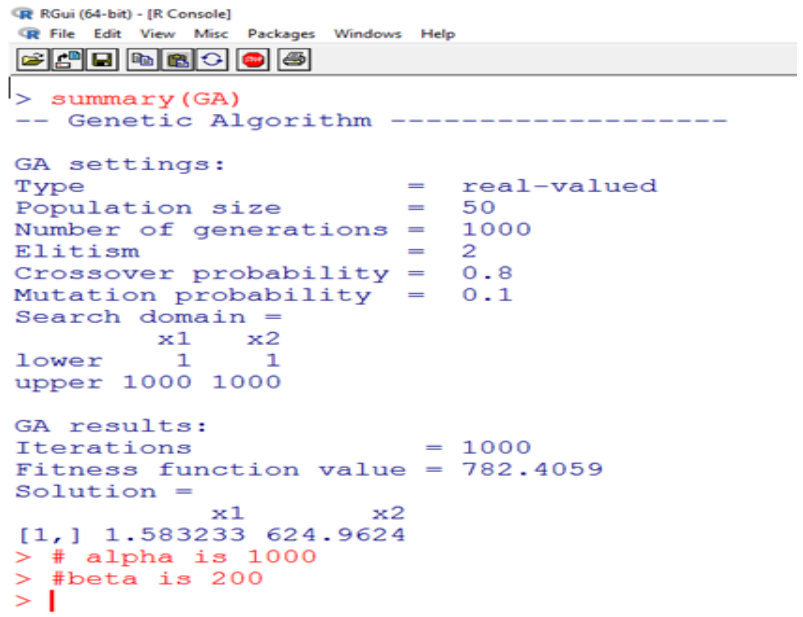

Figure 14: The entire summary of results after running the code.

The Figure 13 shows how the fitness varies as the generation are increased correspondingly and the figure 14 shows result as well as the various parameters with which this genetic algorithm is run and with the fact that we want to use less amount of inputs to get maximum output keeping the constraints in mind.The results that we got are:

$\mathrm{n}=624$

$\mathrm{P}=1.58$ watt

Thus during practical design of the system, we should keep the above parameters in mind. 


\section{CONCLUSION}

The system that we have proposed utilizes effectively the waste energy, by utilizing this system a considerable amount of money can also be saved which can benefit the society as well as improve the GDP of the country. Thus this system that is developed in this paper is an efficient means of conversion of energy as it does not depends verily on climatic conditions like other renewable resources that are presently in use today depends. Further scope for development of this paper could be making an automatically orient able shaft that adjusts its orientation of the wind power generator depending on the amount of power that is developed.

\section{ACKNOWLEDGMENTS}

Our thanks to the experts Dr(Prof) Manas Kumar Sanyal, University of Kalyani, who have advised and encouraged us for such kind of development. In addition, special thanks to Management, JIS College of Engineering, JIS GROUP for providing all kinds of required resources.

\section{REFERENCES}

[1] Md. MoyeedAbrar,"Power cut off and Power Blackout in India a Major threat- An Overview",International Journal of Advancements in Research \& Technology, Volume 5, Issue 7, July-2016, ISSN 2278-7763

[2] Chakraborty, S., Kumar, R., Haldkar, A.K. et al,"Mathematical method to find best suited PV technology for different climatic zones of India", Int J Energy Environ Eng (2017) 8: 153, https://link.springer.com/article/10.1007/s40095-016$0227-\mathrm{z}$

\section{AUTHOR'S PROFILE}

Sumit Das is presently working as an Asst. Professor in the Department of Information Technology, JIS College of Engineering, West Bengal, India. He completed his M.Tech degree in Computer Science and Engineering from University of Kalyani in the year 2008.

Dr (Prof) Manas Kumar Sanyal is presently working as a Professor in the Department of Business Administration, former HOD, and Dean, faculty of Engineering, Technology and Management (ETM), University of Kalyani, West Bengal, India.

Debamoy Datta is a student of 3rd year in Electrical Engineering Department in JIS College of Engineering, West Bengal, India.
[3] Chen, N., Wang, Q., Yao, L. et al, "Wind power forecasting error-based dispatch method for wind farm cluster", J. Mod. Power Syst. Clean Energy (2013), https://link.springer.com/article/10.1007/s40565-0130004-4

[4] Lu, W., \& Roberts, A. (2011). U.S. Patent No. US8067878B1. Washington, DC: U.S. Patent and Trademark Office.

[5] SlabochPaul,"An Operational Model for the Prediction of Jet Blast", January 2012,DOI: 10.2514/6.20121229,Conference: 50th AIAA Aerospace Sciences Meeting including the New Horizons Forum and Aerospace Exposition.

[6] Wikipedia contributors. (2018, June 1). Frequency changer. In Wikipedia, The Free Encyclopedia. Retrieved 03:40, October 8, 2018 , from https://en.wikipedia.org/w/index.php?title=Frequen cy_changer\&oldid $=843916439$

[7] Luca Scrucca,"GA: A Package for Genetic Algorithms in R",Journal of Statistical Software,April 2013, Volume 53, Issue 4, http://www.jstatsoft.org

[8] S Das et al,“A Novel Design of Super-Capacitor Used To Enhance Solar-Energy Restoration of Photo-Voltaic Cells”, ICONCE 2014, January 16 - 17, 2014, Kalyani, WB, India, PRINT ISBN 978-1-4799-3339-6, ISBN 978-1-4799-3338-9/14/\$31.00 @2014 IEEE

[9] S Das et al"An Integrated Design of an Auto Clean and Cooling Smart PV Panel", International Journal of Innovations in Engineering and Technology (IJIET), Vol. 4 Issue 1 June 2014, ISSN: 2319 - 1058 